Fig. 1. Querschnitt einer Falte aus dem menschlichen Jejunum. Gezeichnet mit: $\mathrm{Z}$ e is s Ob. E. Oc. II Camera.

Fig. 2. Längsschnitt einer Falte des menschlichen Jejunum. Gez. mit: $\mathrm{Z}$ e is s Hom. Imm. $1 / 18$, Oc. II Camera.

Figg. 3-11. Epithelzellen aus dem Jejunum des Menschen mit angrenzenden Partien der Tunica propria. Zeiss Hom. Imm. 1/18, Oc. II Camera.

Fig: 12. Abschnitt aus dem Boden einer Krypta des Processus vermiformis des Meerschweinchens. Gez.: Zeiss. Hom. Imm. 1/18, Oc. II Camera.

Fig. 13. Dasselbe. 1, 2, 3, 4 Uebergangsformen der epithelialen Kerne in Leucocytenkerne.

Fig. 14. Senkrechter Schnitt durch eine Krypta des Processus vermiformis des Meerschweinchens. Gez. mit: Ze is s BB, Oc. II. Camera. $\mathrm{CBz}=$ comprimirte Becherzelle.

Figg. 15, 16 und 17. Epithelzellen aus dem Epithel der Wand der Krypta aus dem Processus vermiformis des Meerschweinchens. Gez. mit: $\mathrm{Z}$ e is s Hom. Imm. $1 / 18$, Oc. II Camera.

\title{
Ueber die Bildung von Bürstenbesätzen an den Epithelien diphtherisch erkrankter Nieren.
}

\author{
Von
}

Prof. Dr. Oertel in München.

Hierzu Tafel XXXII.

Die Beobachtungen über das Auftreten von Bürstenbesätzen an Driisenepithelien theils unter physiologischen, theils unter pathologischen Verhältnissen haben sich in der letzten Zeit ausserordentlich vermehrt, ohne dass es bis jetzt möglich gewesen wäre, ein bestimmtes Urtheil iuber die Bedeutung derselben zu gewinnen.

Den in den vorausgehenden Heften dieses Archivs veröffentlichten Untersuchungen über jene merkwürdigen Bildungen möchte ich eine neue Beobachtung anreihen, die rein nur auf pathologische Vorgänge bezogen werden kann, und manche Eigenthümlichkeiten 
bietet, durch welche sie sich insbesondere von anderen Beobachtungen unterscheidet.

$\mathrm{Da}$ schon in den beiden vorausgebenden Aufsätzen von 0 . Tornier ${ }^{1}$ ) und Joh. Frenzel ${ }^{2}$ ) die vorliegende Literatur so ziemlich vollständig angegeben ist, so kann ich wohl von der wiederholten Aufführung derselben Umgang nebmen. Ich reibe demnach meine Beobachtungen einfach den dort verzeichneten pathologischen Vorgängen von Erkrankung der Nieren nach akut verlaufender Phosphorvergiftung, Eklampsie, Vergiftung eines Hundes durch chlorsaures Kali und darauffolgender Hämoglobinurie an, und füge diesen nur noch bei, dass Hen eage Gibbes ${ }^{3}$ ) Bursten- oder Cilienbesätze in zahlreichen IIarnkanälchen bei perniciöser, progressiver Anämie, und Professor Tuttle ${ }^{4}$ ) in Ohio dieselben auch bei Pocken (Smallpox) aufgefunden hat. Meine eigenen Beobachtungen erstrecken sich auf mehrere akut verlaufende Fälle von schwerer croupöser und septischer Diphtherie. Chlorsaures Kali wurde nach ärztlicher Aussage nur in schwacher Lösung als Gurgelwasser benutzt. Hämoglobinurie war in keinem Falle, weder im Leben noch in den Veränderungen der der Obduktion entnommenen Nieren bei sorgtältigster Prüfung nachzuweisen.

In zehn Nieren, welche ich einer eingehenden mikroskopischen Analyse unterwarf, und dazu immer grössere Serien von Schnitten benutzte, fand ich dreimal nachfolgende Veränderungen der Epithelien in den gewundenen und geraden Harnkanälchendes Labyrinths.

-Während in der Mehrzahl die Erscheinungen der akuten parenchymatösen Glomerulo-Nephritis vorlagen mit mehr oder weniger weit vorgeschrittener Nekrose der Epithelien in den Tub. contort. et rect., war in drei Nieren entweder nur spärlich, in einem Dutzend von Schnitten vielleicht nur in einem einzigen Kanälchen, oder aber unter Zurücktreten der einfachen Nekrose in überwiegender Zahl, oder fast ausschliesslich eine Erkrankung der

1) 0. Tornier, Ueber Bürstenbesätze an Drüsenepithelien. Arch. f. mikr. Anat. Bd. 27, H. III, S. 181.

2) J. Frenzel, Zum feineren Bau des Wimperapparates. Arch. f. mikr. Anat. Bd. 28, p. 74 etc.

3) Heneage Gibbes, Quarterly Journal of microscopal science. Bd. 24. Nene Folge. 1884, p. 192.

4) In dem vorher citirten Aufsatze von $\mathrm{H}$. Gibbes mitgetheilt. 
Epithelien zu constatiren, welche zu eigenthümlichen Veränderungen und theilweiser Auflösung derselben führte.

Als erste Erscheinungen an solchen Epithellzellen sah man dass der convexe Rand des in das Lumen der Kanälchen einspringenden Fortsatzes der entzündlich vergrösserten Zelle seine gewöhnliche Begrenzung, oder wenn wir sagen wollen, Zellmembran verlor, und entsprechend dem bogenförmigen Contour eine Reihe von Stäbchen erkennen liess, welche wie Cilien der Zelle anfsassen, und unter Benutzung der apochromatischen Linse von $Z$ e iss, homog. Immers. Ob. $2,0 \mathrm{~mm}$ und Oc. $25 \mathrm{~mm}$, eine ziemlich breite und knorrige Form zeigten. Die Länge der einzelnen Stäbchen war entweder fast allenthalben die gleiche, oder die mittleren, welche am Gipfel lagen, waren etwas grösser, während die seitlichen sich mehr oder weniger verkürzten. Die Stäbchen selbst standen in solchen Zellen immer noch weit über dem Kerne, am höchsten, entsprechend der konischen Form der Zelle, in der Mitte, und eine an ihrer Basis gedachte Linie beschrieb einen starken, convexen Bogen über dem Kerne. Die Oberfläche, auf welcher die Stäbchen anfsassen, zeigte indess keine regelmässige Begrenzung, and namentlich war kein schärferer Contur, auch nicht mit stärkeren Linsen, nachweisbar. Die Stäbchen gingen entweder einfach in die Zellsubstanz über, und da die Zwischensubstanz zwischen zwei Stäbchen manchmal etwas weiter hinaufreichte oder weiter hinab fehlte, so war die Begrenzung an der Basis der Stäbchen eine unregelmässige geworden. Dagegen liess sich bei anderen Zellen, und namentlich bei den gleich $\mathrm{zu}$ beschreibenden, immer eine scharf abgrenzende Linie oder Leiste erkennen. In der übrigen Substanz der Zelle selbst war keine stäbchenförmige Bildung im Protoplasma oder eine Längsstrichelung unterscheidbar, sondern dieselbe sah gleichförmig körnig aus, und zeigte nur an einzelnen Stellen durch Auseinanderweichen des Protoplasmas infolge von nekrotischem Zerfall grössere oder kleinere Spalten und Licken.

Waren nun die Veränderungen in solchen Zellen weiter vorgeschritten, so reichten die Stäbchen meist bis in die Nähe des Kernes herab, so dass die Zelle fast halbirt erschien, und man einen protoplasmahaltigen Zelltheil mit dem Kerne und die über demselben liegende Stäbchenschichte unterscheiden musste (Fig. $2 \mathrm{u}$. 3). Die Stäbchen selbst zeigten an den so veränderten Zellen fast 
allenthalhen die gleiche Grösse, gingen aber jetzt nicht mehr unmittelbar in die Zellsubstanz über, sondern wurden von derselben durch eine deutlich erkennbare Zwischenleiste (Fig. 2 u. 3 Qu. L.) getrennt, auf der sie aufsassen, oder mit der sie ohne Differenzirung zusammenhingen. Der unter dieser Leiste liegende Zelltheil liess gleichfalls wieder je nach dem Fortschritt der Erkrankung eine verschiedene Anordnung seiner Zellsubstanz erkennen. In den einen Zellen verbielt sich die Zellsubstanz vollkommen gleich wie in den zuerst beschriebenen Zellen, erschien als feinkörnige Masse von verschiedener Dichte, aber in unregelmässiger Anordnung und durch nekrotische Lücken und Spalten auseinandergerissen. (Fig. $2 \mathrm{~N}$. S). In anderen Zellen dagegen waren unter der horizontal verlaufenden Leiste ebenfalls stäbchenförmige Gebilde erkennbar, welche von dieser bis zur Basis der Zelle binabreichten, in dichten Reihen zwischen den meist weit auseinanderliegenden Kernen standen und zwischen sich eine feinkörnige Substanz erkennen liessen, während die über der Leiste stehenden Stäbchen entweder keine solche zwischen sich einschlossen und vollkommen isolirt erschienen, oder nur mehr wenige Körnchen in einfacher oder dünner Lage bargen (Fig. 4 O. St. u. U. St.). Durch diese morphologischen Veränderungen war auch das optische Verhalten der beiden Schichten ein vollkommen verschiedenes gevorden. Während die obere Schichte weniger stark lichtbrechend nur in feinsten Linien ibre Elemente erkennen liess, war die untere undurchsichtiger und mehr körnig getrübt, so dass die Contouren der einzelnen Bestandtheile nicht so deutlich heraustraten und fitr das Auge isolirbar waren. Die Begrenzungslinie der beiden Schichten war durch die Leiste eine ziemlich scharfe, und bei genauer Einstellung bekam man den Eindruck einer senkrecht zum Bilde stehenden Fläche, auf welcher die Stäbchen so inserirten, dass auf der Schnittfläche noch eine schmale Kante frei blieb und auf diese Weise eine scharf gezogene Linie bildete. Die von Tornier und anderen beobachtete schwarze Begrenzungslinie diurte mit der in meinen Präparaten beobachteten Leiste wohl identisch sein. Die Höhe der Leiste über der Basalfäche der Zelle war überall die gleiche, etwas grösser als die Kernhöhe, so dass der noch restirende Zellbelag im Kanälchen überall die gleiche Höbe einhielt. Dadurch ersehienen auch die Harnkanälchen immer noch mit einem gleichförmigen Epithelring ansgekleidet, bei welchem nur 
die Zellen 1/2 mal so hoch waren als im Normalen (Fig. 1 u. 4). Der feblende Theil der Höhe der gewöhnlichen Epithelzellen wurde annähernd durch einen Saum von feinen, stäbchenartigen Gebilden ergänzt der auch bei schwacher Vergrösserung den Eindruck eines Cilien- oder Bürstenbesatzes bervorbrachte.

Was nun das durch die Reduktion der Epithelien erweiterte Lumen der Harnkanälchen anbelangt, so war in demselben immer eine grössere oder geringere Menge kleinster Körnchen enthalten, die verschiedenartig gruppirt waren und vollkommen jenen glichen, welche zwischen den einzelnen sowohl freien wie im unteren Theile der Zellen liegenden Stäbchen eingeschlossen waren. Ihre Lagerung über den Stäbchen war manchmal derart in Reihen geordnet, als wären sie so unmittelbar aus den Stäbchen herausgefallen.

Suchen wir nach einer Erklärung dieser Veränderungen an den Epithelien der Tub. contort. et rect., so werden wir wohl unter den vorwaltenden Umständen von funktionellen Veränderungen ganz und gar absehen und sie vielmehr als rein pathologische durch eine Beschädigung der Zellen veranlasste auffassen müssen. Die unmittelbare Ursache liegt zweifellos in der Aufnahme eines Giftes, welehes die Erkrankung und theilweise Auflösung der Zellen zur Folge gehabt hat, und speciell in unserem Falle in der Infektion der Zellen durch das diphtherische Gift am wahrscheinlichsten zu suchen ist. Wie das diphtherische Gift in den anderen Harnkanälchen eine totale Nekrose der Zellen und körnigen Zerfall durch stärkere Einwirkung hervorgebracht hat (Fig. 1), so ist es in einer Reihe von Kanälchen möglicherweise durch eine abgeschwächte Wirkung desselben nur zu einer theilweisen Zerstörung gekommen. Die Zellmembran oder dichtere Begrenzungsschichte des Fortsatzes der Epithelien wurde aufgelöst, und der zwischen den Stäbchen der Zelle oder der stäbchenförmigen Anordnung des Protoplasma's gelegene körnige Inhalt, das Paraplasma im Sinne K upffer's, entleerte sich in das Innere des Kanälchens. Die Verschiedenheit unter den folgenden Bildern erklärt sich damit einfach aus der Grösse des Zerfalls oder vielmehr der Menge der ausgetretenen körnigen Masse oder des Paraplasmas. Nachdem die obere feste Begrenzungsschichte des Zellfortsatzes einmal aufgelöst war, erfolgte die Entleerung der Stäbchenschichte über der horizontalen Leiste in kürzerer Zeit, so dass nur mehr wenige 
Zellen noch zu finden waren, in welchen kurze, stäbchenartige Fortsätze unmittelbal in die Zellsubstanz übergingen, während auf weite Strecken des Labyrinths hin in allen Zellen der Kanälehen der Körnerausfall bereits bis auf die horizontale Leiste sich erstreckt hatte. Zweifellos wird durch diese Leiste, für deren stïndiges Vorhandensein. auch die gleichmässig eingehaltene Höhe des $z \mathfrak{u}$ rückgebliebenen Zellenringes spricht, das weitere Austreten der Körnchen aus den tieferen Partien der Zellen entweder für einige Zeit noch vollsändig verhindert, wie die Präparate zeigen, in welchen die untere Zellpartie einfach körnig erscheint, und keine stäbchenförmige Differenzirung in seiner Substanz erkennen lässt, oder der Ausfall der Körnchen durch dieselbe erfolgt nur langsam and allmählich unter deutlicherem Hervortreten der Stäbchen in der lichter werdenden Körnermasse.

Schliessen wir aus dem Ausfall der Körnchenmasse oder des Paraplasmas der Epithelien auf die Beschaffenheit der Zwischenleiste, so miissen wir uns dieselbe als eine poröse, vielfach durchlöcherte, in ihren einzelnen Bestandtheilen indess festgefügte, dïnne Schichte, vielleicht von derselben Substanz wie die Stäbchen, vorstellen, in welche sowohl die oberen wie die unteren oftmals direkt überzugehen schienen, und die durch die allenthalben vorhandenen Lücken das allmähliche Ansfallen der unteren Körnermasse noch gestattet. Wir könnten aber auch annebmen, dass die Zwischenkörnersehichte an dieser Stelle überhaupt nur fester gefügt ist, als in den darüber oder darunter liegenden Theilen der Zelle. Damit kämen wir indess auf die gleiche Anschaung hinans; die fester gefligte Körnerlage müsste immer ein Hindurchtreten der unteren Körnermasse gestatten, ohne selbst eine Beschädigung zu erfahren; denn man kann nicht beobachten, dass an der einen oder anderen Stelle mehr Körner zwischen den Stäbchen herausfallen, und die Begrenzung der unteren Zellsubstanz dadurch gezackt oder eingekerbt aussäihe, sondern in allen Harnkanälchen, und selbst tiber weite Strecken des Labyrinths hin wird eine und dieselbe Höhe der restirenden Zellen eingehalten und durch eine bei starker Vergrösserung deutlich sichtbare Leiste begrenzt. Dieselbe Grenze der degenerirten Zellen wird endlich auch noch eingehalten, wenn die Stäbchen bei fortschreitenden Veränderungen za Grunde gegangen oder abgefallen sind, und der noch zurückgebliebene Zellring entweder zu einem Epithel- 
cylinder sich zusammenschliesst, oder in das Lumen desselben eine gerinnfähige Substanz austritt und einen Fibrincylinder bildet. Ich werde auf diese Vorgänge an einem anderen Orte noch eingehend zu sprechen kommen ${ }^{1}$ ).

Wenn wir nun die obigen Beobachtungen vergleichen mit den von Tornier beschriebenen, bei welchen er nach reiflicher Erwägung aller Umstände das Auftreten der Bürstenbesätze als eine physiologische Erscheinung, die an bestimmte funktionelle, beziehungsweise sekretorische Vorgänge in der Zelle gebunden ist; erklären musste, so werden wir in unserem Falle das Freiwerden sowohl der oberen wir der unteren Stäbchenschichte an den Epithelien der Harnkanälchen als einen theilweisen'Zerfall der Zellen bezeichnen müssen, der mit dem vollständigen Zugrundegehen der Zelle selbst endigt. Ein beachtenswerther Unterschied liegt ferner in der grosen Verschiedenheit der Art und Weise, wie die Stäbchenschichte oder die Bürstenbesätze der Zellen der Beobachtung sich erschlossen. Während Tornier nur an Präparaten, welche frisch dem Thiere entnommen und in Sublimatlösung (Marchaud benutzte Osmiumsäure) erhärtet waren, nachweisen konnte, wurden die mir vorliegenden Präparate frühestens 10, in der Regel 12 bis 18 Stunden nach dem Tode der Leiche entnommen und einfach in Alkobol erbärtet, und entweder mit Fuchsin oder Hämatoxylinkarmin gefärbt, wobei mit letzterer Farbe eine schwach röthliche Tinktion der Stäbchen und Zellsubstanz erzielt wurde, während die Kerne sich violett färbten. Das rasche Verschwinden der von verschiedener Seite beobachteten Bürstenbesätze diurfte vielleicht in dem raschen Wechsel der physiologischen Vorgänge in der Zelle und ihrer Substanz während der Verdaung oder Sekretion begründet sein, indess hier durch Aufösung der Greuzschichte die Zwischenkörnerlage, das Paraplasma, einfach austrat und die Stälchen sichtbar werden liess. Diese selbst blieben so lange erhalten, als in der Zelle noch keine neuen pathologischen Erscheinungen auftraten, und stiessen sich erst $a b$, wenn die Dekomposition der Zellen weiter fortschritt.

Da nun Tuttle dieselben Veränderungen der Niere bei Blattern gefunden, in den vorliegenden drei Fällen jedesmal eine

1) In einer grössern demnächst dem Druck zu übergebenden Arbeit über die Pathogenese der Diphtherie. 
532 Dr. Oertel: Ueber d. Bildung von Bürstenbesätzen an den Epithelien etc.

schwere diphtherische Infektion bestanden bat, wobei in einem Falle chlorsaures Kali weder als Gurgelwasser noch innerlich verabreicht wurde, noch überhaupt ein Medikament ${ }^{1}$ ), auf welches eine Nierenreizung eintreten kann, so erübrigt zu ihrer Erklärung nur mehr, dass die Auflösung der Zellen durch das bei diesen Infektionskrankheiten eigenthümliche Gift selbst hervorgebracht wird, das, von den Nierenepithelien aufgenommen, eine Destruktion derselben zur Folge hat. Es wird sich nun fragen, wie weit diese Veränderungen noch bei anderen Infektionskrankheiten und Vergiftungen sich finden. Von grossem histologischen Interesse ist die durch diese Auflösung der Epithelzellen in den gewundenen and geraden Harnkanälchen bedingte Darlegung ihres Aufbaues und ihrer einzelnen feineren Bestandtheile, und die dadurch auf pathologischem Wege gelieferte Bestätigung von der Richtigkeit der vorausgegangenen anatomischen Forschung.

\section{Erklärung der Abbildungen auf Tafel XXXII.}

Fig. 1. In nekrotischem Zerfall begriffenes Epithel eines Harnkanälchens aus dem Labyrinth. EZ. = Epithelzelle. Zeiss Ob. E, Oc. IV.

Fig. 2. Epithelzellen, deren obere Partie in Auflösung begriffen, stäbchenförmige Bildungen zeigt. 0 . St. = obere Stäbchen; Qu. L. = Querleiste; N. S. = nekrotische Substanz der unteren Partie ler Zelle. $\mathrm{Z}$ e is $\mathrm{s}$ A pochrom. Linse, homog. Immers. Ob. $2,0 \mathrm{~mm}$, Oc. $25 \mathrm{~mm}$, Tubuslänge $160 \mathrm{~mm}$.

Fig. 3. Derselbe Process. O. St. = obere Stäbchen; Qu. L. = Querleiste. P. S. = zwischen den Stäbchen ausgetretene paraplasmatische Substanz. Vergr. wie in Fig. 2.

Fig. 4. Querschnitt eines gewundenen Kanälchens, in welchem die Epithelien durch die ganze Auskleidung hindurch stäbchenförmige Bildungen erkennen lassen. 0 . St. $=$ Stäbchen am oberen, U. St. $=$ Stäbchen am unteren Theile der Zellen sichtbar geworden. Qu. L. = Querleiste. P. S. = zwischen den Stäbchen ausgetretene paraplasmatische Substanz. Zeiss Ob. E, Oc. IV.

1) Die Fälle wurden von mir nicht selbst behandelt, sondern die Medikamentation mir nur nachträglich im Allgemeinen bekannt gegeben. $\mathrm{Ob}$ in den beiden andern Fällen die Nierenveränderungen zum Theil auch durch kleinere Mengen der beim Gurgeln etwa unwillkürlich verschluckten 2-3\% chlorsauren Kalilösung verursacht wurden, muss ich unentschieden lassen. 


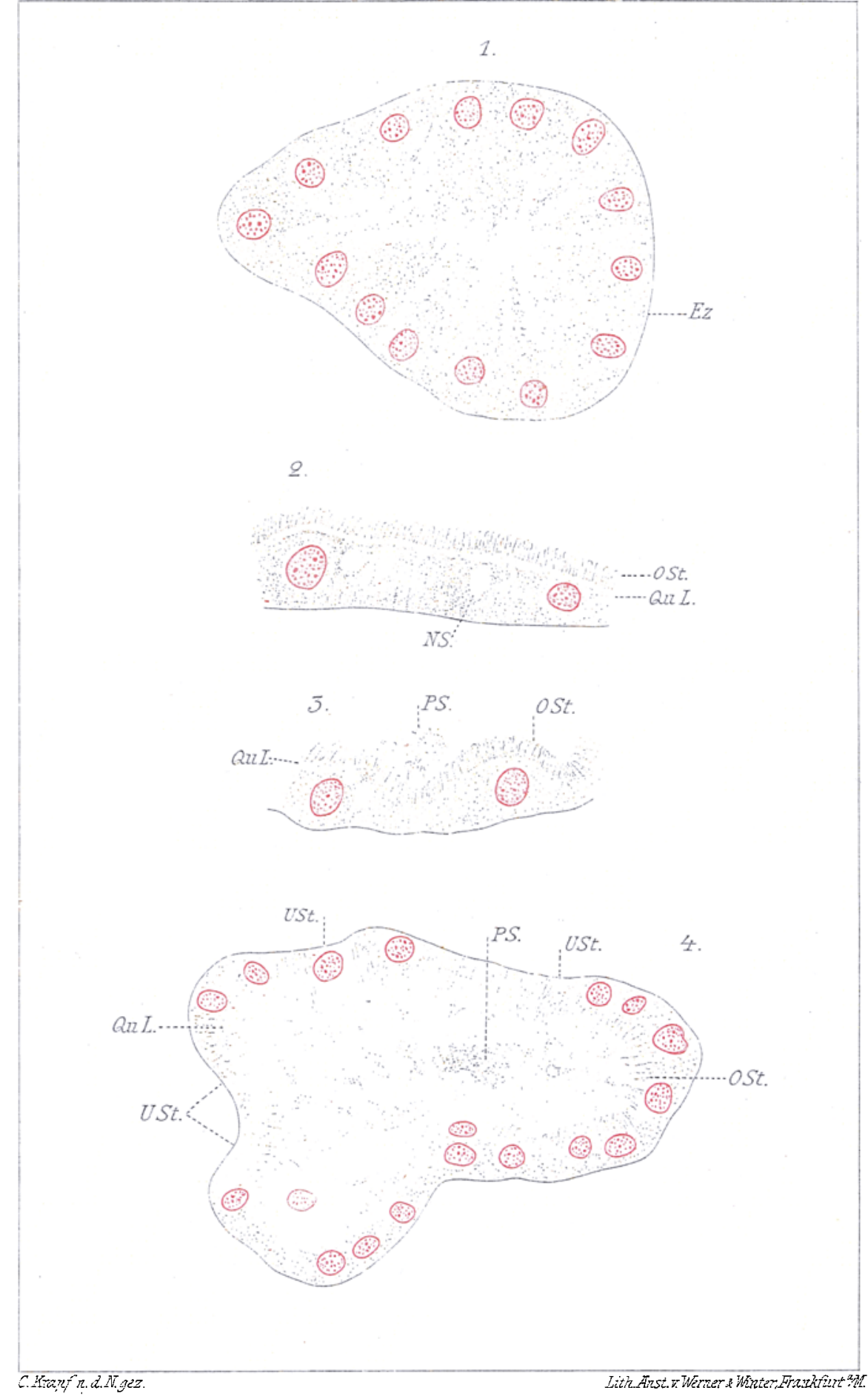

